\title{
Scattering of thermal He beams by crossed atomic and molecular beams. I. Sensitivity of the elastic differential cross section to the interatomic potential ${ }^{\mathbf{a})}$
}

\author{
Mark Keil ${ }^{\text {b) }}$ and Aron Kuppermann \\ Arthur Amos Noyes Laboratory of Chemical Physics, ${ }^{c}$ California Institute of Technology, Pasadena, \\ California 91125 \\ (Received 20 December 1977) \\ The ability of diffraction oscillations in atomic beam scattering experiments to uniquely determine \\ interatomic potentials for highly quantal systems is examined. Assumed but realistic potentials are used to \\ generate, by scattering calculations and incorporation of random errors, differential cross sections which \\ are then treated as if they were "experimental" data. From these, attempts are made to recover the \\ initial potential by varying the parameters of assumed mathematical forms different from the original one, \\ until a best fit to the "experimental" results is obtained. It is found that the region of the interaction \\ potential around the van der Waals minimum is accurately determined by the "measured" differential \\ cross sections over a range of interatomic separations significantly wider than would be expected \\ classically. It is also found, for collision energies at which the weakly repulsive wall is appreciably \\ sampled, that the SPF-Dunham and double Morse-van der Waals types of potentials lead to accurate \\ determinations of the interatomic potential, whereas many other mathematical forms do not. Analytical \\ parameterizations most appropriate for obtaining accurate interatomic potentials from thermal DCS \\ experiments, for a given highly quantal system, may depend on the collision energy used.
}

\section{INTRODUCTION}

In recent years, interatomic potentials between closedshell species have become better determined. ${ }^{1-3}$ However, the important region of the van der Waals attractive minimum results from the balance between the competing short- and long-range forces, making its theoretical characterization more difficult than that of the adjacent regions." Various experimental techniques have also been used to obtain potentials with increased reliability over specific ranges of interatomic separations. Recently, a more unified approach has been adopted in the use of various types of experimental data to determine a potential curve describing the interaction over a wide range of separations. ${ }^{5}$ The suitability of virial ${ }^{6,7}$ and transport coefficient ${ }^{50,7,8}$ data and of integral cross sections ${ }^{7 \mathrm{~b}, 9}$ for the determination of the potential has been examined elsewhere. ${ }^{x, 10}$ In this paper we extend this examination to differential cross section (DCS) scattering data - exhibiting rapid quantum oscillations and sometimes also a vestige of the classical rainbow - which are widely regarded as a means of probing the attractive well and low-energy repulsive regions. 24,11 . The sensitivity of such DCS data to the interatomic potentials for highly quantal systems may be significantly different from that indicated by classical or semiclassical considerations, ${ }^{3 f}$ and heretofore has not been examined in detail. In a subsequent paper ${ }^{12}$ (hereafter referred to as Paper II of this series), we will analyze the results of our own recent measurements of the He-Ar DCS, in light of the present discussion.

Although the He-Ar system has been studied previ-

\footnotetext{
${ }^{2}$ This work was supported in part by a contract (No. EY-76S-03-767) from the Department of Energy. Report Code: CALT-767P4-151.

b) Work performed in partial fulfillment of the requirements for the Ph. D. in Chemistry at the California Institute of Technology.

${ }^{c)}$ Contribution No. 5714 .
}

ously by differential scattering, ${ }^{13,14}$ as well as by a wide variety of other techniques, ${ }^{13}$ considerable differences remain in the resulting potentials. In Sec. II we discuss the limitations that many of these techniques have in determining interatomic potentials for light systems. We also describe several parameterized potentials most commonly used for fitting experimental results which are sensitive to van der Waals interactions. In Sec. III we use realistic interaction potentials (corresponding closely to, e.g. , the He-Ar system) to calculate simulated "experimental" DCS's, to which the model potentials of Sec. II are fit. We then compare the "known" potentials with the fitted ones and discuss the results in terms of these various potential forms' flexibility in fitting the DCS data, as well as the accuracy with which each of these forms reproduce the "correct" potentials. By additionally simulating "experimental data scatter" in the DCS's, we also determine the accuracy with which each of the various stylized potentials may be obtained. We concur with Bickes and Bernstein ${ }^{15}$ that the SPFDunham (SPFD) potential ${ }^{16-19}$ is a simple analytical representation that can be used to reproduce the potential and can easily be used for the analysis of various kinds of experimental data. ${ }^{15}$ In Sec. IV we extend (for light systems) their examination of the SPFD potential form, demonstrating the uniqueness of the potential as obtained by an improved regression procedure. We similarly analyze the suitability of a new double Morse-van der Waals ( $\left.\mathrm{M}^{2} \mathrm{SV}\right)$ type of mathematical parameterization, which has a flexibility comparable to that of the SPFD potential form. We further determine the range of interatomic separations to which the DCS data are sensitive. This range should also be applicable to He-molecule systems, and useful in the interpretation of $\mathrm{H}_{2}\left(\mathrm{D}_{2}\right)$ molecule data. ${ }^{20}$ In addition, we analyze the degree to which a given region of the potential may be varied independently of the remaining regions by appropriate changes in the SPFD parameters. We use these results to correlate features of the DCS with particular regions 
of the potential, and to place "confidence levels" on potentials determined by regression techniques from DCS experiments. Finally, we summarize our major conclusions in Sec. V.

\section{INTERATOMIC POTENTIALS}

With a few notable exceptions, ${ }^{3 b, 21,22}$ experimental attempts to determine the weakly attractive van der Waals region of interatomic potentials suffer from a number of difficulties. Foremost among these is the fact that the dependence of measurable properties on the potential is through an integral relation. We first discuss these exceptions.

Direct inversion of molecular beam scattering data has been used in different ways ${ }^{21}$ to obtain potentials for the highly repulsive short-range ("classical") interactions, and in the attractive well region for systems of a "semiclassical" nature. However, because of the large quantum effects for thermal He scattering due to a low $B$ parameter, ${ }^{23}$ these analysis techniques are inappropriate. Quantum mechanical inversion procedures ${ }^{2 a, b}$ are only now beginning to be applied to experimental scattering data. ${ }^{24,25}$

Spectroscopic studies using the RKR method have enabled accurate information to be obtained about the shape of the potential well of rare gas van der Waals molecules supporting significant numbers of bound vibrational states. ${ }^{22}$ Unfortunately the potential energies at which the well width may be evaluated are restricted to the positions of these states. ${ }^{26}$ It is thus possible to obtain only a small amount of information about the potential of most $\mathrm{He}$-containing pairs by this procedure. ${ }^{27}$

Besides these experimental techniques, semiempirical theoretical methods have been used to calculate reliable interatomic potentials for some systems. Most early studies were restricted to short- ${ }^{3 a}$ or long-range ${ }^{28}$ interactions, but detailed results for the intermediate range including the potential minimum are currently available. ${ }^{29-31}$ Although successfully applied to heavy rare gas pairs, $\mathrm{He}$-(and to a lesser extent $\mathrm{Ne}-$ ) containing systems have often been regarded as "anomalous" from the point of view of electron gas theories. ${ }^{29}$ Accurate $a b$ initio results have been obtained for $\mathrm{He}_{2},{ }^{32}$ but such calculations for asymmetric He pairs are still too difficult.

Thus, the above techniques have been exploited profitably, but are still inappropriate for the He-Ar system, herein used as an example for similar systems. Other means must therefore be used to deduce these potentials. A common procedure, which is used to analyze transport and virial coefficient data or low-energy DCS data, is to assume a functional form for the interaction potential and then optimize its parameters to fit the experimental results. Among the possible disadvantages of this method are the lack of uniqueness of the resulting potential, ${ }^{33}$ and the restriction of the potential obtained from the experiment to the assumed form. Furthermore, there is no point-to-point correspondence between the derived potential and the measurements (i.e., they are related by integral expressions), making it difficult to determine the sensitivity of the potential to the data.
To some extent all these difficulties may be addressed by the use of different functional forms for different refions of the potential, which are joined smoothly. This procecure has already been used extensively for rare gas atom-atom scattering data. ${ }^{2 a, 34}$ Another approach is to use a multiparameter functional form with sufficient flexibility to enable the potential to be changed in only one region at a time. ${ }^{2 a}$

Of course, the flexibility that justifiably may be used is limited by the variety and accuracy of the available data, and by its sensitivity to the potential. With the extensive compilations of data that are available for $\mathrm{Ar}_{2}$, it is possible to construct a numerical potential by a multivariate technique. ${ }^{7 a}$ Because such an abundance of data is not available for most systems, our goal, for the analysis of low-energy DCS data, is to examine the suitability of some of the more common functional forms, ${ }^{35}$ each with only a few adjustable parameters. We also compare ${ }^{36}$ these potentials with some of the less flexible but more traditional ones. ${ }^{1}$ All of these are of the "corresponding-states" form, ${ }^{3 \mathrm{~h}}$ with

$$
V(r)=\epsilon f(\rho), \quad \rho=r / r_{m},
$$

where $r_{m}$ and $\epsilon$ are the potential minimum position and depth, respectively; the position of the zero of the potential $\sigma$ may be related to $r_{m}$ and the potential shape $f(\rho)$.

The potential shapes we consider are

a) Kihara, ${ }^{1,3 d, g}$

$$
f(\rho)=\frac{6}{n-6}\left[\left(\frac{1-\gamma}{\rho-\gamma}\right)^{n}-\frac{n}{6}\left(\frac{1-\gamma}{\rho-\gamma}\right)^{6}\right],
$$

where $\gamma$ is the hard-core radius parameter and $n$ is the repulsive term exponent. The Lennard-Jones (LJ) $n-6$ potential may be recovered by setting $\gamma=0$. Note that for $\gamma \neq 0$ the asymptotic behavior includes $\rho^{-7}$ and higherorder terms.

b) Buckingham (exp-6), ${ }^{1,3 \mathrm{~d}, \mathrm{~g}}$

$$
f(\rho)=\frac{1}{\alpha-6}\left[6 e^{\alpha(1-\rho)}-\alpha \rho^{-6}\right] .
$$

The only shape parameter available is $\alpha$, governing the behavior of the exponential repulsion term.

c) Klein-Hanley, ${ }^{37}$

$$
f(\rho)=\frac{\rho^{-n}}{n-8}\left(8-2 c_{6}\right)-\left[c_{6} \rho^{-6}-\frac{\rho^{-8}}{n-8}\left(6 c_{6}+n-n c_{6}\right)\right] .
$$

Shape parameters designed to primarily govern the short- and long-range regions are $n$ and $c_{6},{ }^{38}$ respectively. The second dispersion term in $\rho^{-8}$ is included, but its coefficient is fixed by the other parameters.

d) Buckingham-Corner (BC), 1

$$
f(\rho)=\frac{e^{b(1 \gamma)}}{b-8}\left(8-2 c_{6}\right)-\left[c_{6} \rho^{-6}+\frac{\rho^{-8}}{b-8}\left(6 c_{6}+b-b c_{6}\right)\right] g(\rho),
$$

where

$$
g(\rho)=e^{-\left[4(1 / \rho-1)^{3}\right]} \text { for } \rho<1 \text { and } g(\rho)=1 \text { for } \rho \geqslant 1 .
$$

As for the Klein-Hanley potential, the shape parameters 
$b$ and $c_{6}$ are designed to govern the short- and longrange regions, respectively. Similarly, the second dispersion term in $p^{-8}$ is included, but its coefficient is fixed by the other parameters. The Tang-Toennies semitheoretical ${ }^{312}$ and Hartree-Fock dispersion ${ }^{31 b}$ models are variants of the $\mathrm{BC}$ potential.

e) Barker-Bobetic-Pompe (BBP), ${ }^{5 e, 39}$

$$
f(\rho)=e^{\alpha(1-\rho)}\left[A_{0}+\sum_{i=1}^{N} A_{i}(\rho-1)^{i}\right]-\sum_{i=1}^{3} c_{2 i+4} \rho^{-(2 i+4)},
$$

with

$$
A_{0}=\sum_{i=1}^{3} c_{2 i+4}-1
$$

and

$$
A_{1}=\alpha A_{0}-\sum_{i=1}^{3}(2 i+4) c_{2 i+4} .
$$

Available parameters are $\alpha$ [bearing some resemblance to the exp- $6 \alpha$ of Eq. (2)], the $A_{i}$ coefficients $(i=2, \ldots$, $N)$, and the dispersion constants $\left(c_{6}, c_{8}, c_{10}\right)$. The expansion order $N$ is usually no larger than 5. This $N$ th order BBP potential is highly flexible and is appealing theoretically, with the dispersion series being added to the exponentially repulsive term.

Although some of the potential energy functions above are rather flexible, they all suffer from the drawback that the long- and short-range behaviors are interdependent. To ameliorate this problem, we also investigated several piecewise analytical potentials with smooth joining conditions.

f) Double Lennard- Jones, ${ }^{40}$

$$
\begin{aligned}
& f(\rho)=\frac{m}{n_{1}-m}\left(\rho^{-n_{1}}-\frac{n_{1}}{m} \rho^{-m}\right) \text { for } \rho<1, \\
& f(\rho)=\frac{6}{n_{2}-6}\left(\rho^{-n_{2}}-\frac{n_{2}}{6} \rho^{-6}\right) \text { for } \rho \geq 1 .
\end{aligned}
$$

Use of the $L^{\top}$ form [Eq. (1) with $\gamma=0$ ] for each branch assures smoothness at $\rho=1$. Available shape parameters are $n_{1}, m$, and $n_{2}$; the last may be used to determine the leading dispersion term independently of the repulsive region.

g) Morse-Spline-van der Waals (MSV) ${ }^{41}$ and $\mathrm{M}^{2} \mathrm{SV}$,

$$
\begin{aligned}
f(\rho)= & e^{\beta(1-\rho)}\left[e^{\beta(1-\rho)}-2\right] \quad \text { for } \rho \leqslant \rho_{1} \\
f(\rho)= & \left(\rho_{2}-\rho\right)\left[s_{1}\left(\rho_{2}-\rho\right)^{2}+s_{3}\right] \\
& +\left(\rho-\rho_{1}\right)\left[s_{2}\left(\rho-\rho_{1}\right)^{2}+s_{4}\right] \text { for } \rho_{1}<\rho<\rho_{2} \\
f(\rho)= & -\sum_{i=1}^{3} c_{2 i+4} \rho^{-(2 i+4)} \quad \text { for } \rho \geqslant \rho_{2} .
\end{aligned}
$$

The $s_{i}(i=1,2,3,4)$ are cubic spline coefficients, which are fixed by smoothness conditions at the spline points $\rho_{1}$ and $\rho_{2}$. The first spline point is taken as the inflection point of the Morse curve, $\rho_{1}=1+\beta^{-1}$ ln 2 . The second spline point may be treated as a fitted parameter but is usually held fixed at $1.6 .^{29 \mathrm{a}}$ Remaining shape parameters are then $\beta$, governing the shape of the repulsive and well regions, and the dispersion series coefficients.
We may modify this potential by using a second Morse function for $r<\sigma$, joined smoothly to the first at $r=\sigma$. This $\mathrm{M}^{2} \mathrm{SV}$ potential then has the additional parameter $\beta^{\prime}$.

h) SPF-Dunham (SPFD),
$f(\rho)=b_{0} \lambda^{2}\left(1+\sum_{i=1}^{N} b_{i} \lambda^{i}\right)-1$, for $\lambda=1-(1 / \rho)$ and $\rho<\rho_{f}$,

$$
f(\rho)=-\sum_{i=1}^{3} c_{2 i+4} \rho^{-(2 i+4)} \text { for } \rho \geqslant \rho_{f} .
$$

Smoothness conditions at $\rho=\rho_{f}$ are used to fix the highest-order coefficients $b_{N}$ and $b_{N-1}$. The joining point $\rho_{f}$ is treated in the same fashion as the second spline point of the MSV form and is normally larger than 1; similarly, the $b_{i}(i=1, \ldots, N-2)$ SPFD coefficients determine the shape of the repulsive and well regions while the dispersion series constants are also independent parameters.

For reduced distances below $\rho=0.65$, the exp-6, BBP, and SPFD potentials often show spurious maxima or oscillations. These are removed by using an exponentially repulsive piece, i. e., $f(\rho)=A e^{\text {thp }}$, from some joining value $\rho_{i}$ downwards. Smoothness conditions at $\rho_{i}$ are used to fix $A$ and $b$. Trial calculations show that values of $\rho_{i}<0.75$ have no effect on the scattering results and we use $\rho_{i}=0.70$.

All these potentials are used for the scattering calculations presented here and some of them are used for the experimental data reduction procedures that are described in detail in Paper II. Care has been taken to perform these calculations with a high degree of accuracy and consistency in order to be able to make distinctions between various closely related interatomic pair potentials. The procedure consists of the standard partial wave summation (using exact quantum or RosenYennie $^{3 c, 42}$ phase shifts for $l<50$, after which JWKB phase shifts are adequate), followed by kinematic transformations to the laboratory frame (using room-temperature hypersonic beams and out- of-plane detection of He atoms). The calculations include extensive velocity and angular averagings. The calculational parameters used are chosen to simulate the experimental conditions of Paper II, which are typical for DCS measurements for highly quantal systems, and are described in detail in that paper. Experimental conditions assumed for this study are a relative collision energy of $64 \mathrm{meV}$, with a corresponding relative velocity resolution of $\sim 11 \%$, and an overall angular resolution of $\sim 2^{\circ}$. The range of scattering angles considered is from $1.5^{\circ}$ to $20^{\circ}$, with intensity measurement errors of $\sim 2.5 \%-\sim 20 \%$, respectively. ${ }^{12}$ The simulation described here enables us to gain detailed insight as to the amount of information that may be extracted from the actual experiments. It further suggests criteria helpful in choosing mathematical parameterizations (in addition to those specifically included in this study) suitable for iteratively determining the van der Waals attractive regions of highly quantal systems. 


\section{POTENTIAL FORM SUITABILITY}

The suitability of a particular functional form chosen to represent the potential must be judged by several criteria. Obviously, it must reproduce experimental data which are sensitive to it. This form should also have sufficient flexibility to approximate closely the true potential, whose mathematical form is usually unknown. Finally, the potential obtained must be unique over the range of interatomic distances for which the data are sensitive. The correspondence to the real interaction of any stylized potential derived from experimental measurements depends directly on the extent to which the experiment and the potential meet the above criteria. Our method of determining this correspondence is to use the potentials of the previous section to fit the scattering results corresponding to an assumed interaction.

As noted in the Introduction, $\mathrm{He}-\mathrm{Ar}$ is an example of a system whose interatomic potential is still the subject of considerable controversy. Because our purpose here is merely to discuss the suitability of the iterative procedures used to determine potentials from scattering experiments, we arbitrarily use as the known potential one which very closely resembles a recently published potential for $\mathrm{He}-\mathrm{Ar}^{13}$ as the "known" interaction. We use it to calculate DCS's and then add appropriate data scatter. Treating these as simulated experimental results, we can then obtain fitted potentials iteratively. The analytical forms used in this procedure are different from that used to represent the "correct" potential, just as in the actual data reduction procedure. This somewhat circular route enables us to determine the suitability of a given functional form according to the first two criteria outlined above. Compliance with the third criterion is later gauged for the particularly successful SPFD functional form [Eq. (8)]. Because the potential governing the interaction is "known," the circular approach is more pertinent to the present discussion than would be comparisons of experimentally determined potentials with one another or with theoretical ones, where none of the potentials are known to be "correct."

We choose two different "known" potentials for the present simulation studies. One is given by the MSV functional form [Eq. (7)], with the following parame$\operatorname{ters}^{13}$ :

$$
\begin{aligned}
& r_{m}=3.540 \AA, \quad \epsilon=2.085 \mathrm{meV}, \beta=5.45, \\
& \rho_{2}=1.40, \quad c_{3}=1.434, \quad c_{8}=0.220, \quad c_{10}=0.00 .
\end{aligned}
$$

It must be emphasized that this potential need not actually be "correct" for the discussion presented here to be valid, nor should one construe any particular significance to the choice of the MSV shape. The other "known" potential is given by the Klein-Hanley functional form [Eq. (3) ], as discussed in Sec. IV.A. Using the experimental conditions of Paper $I$, the MSV reference potential was used to calculate a DCS versus scattering angle curve (points exactly on the solid curve of Fig. 1), which then serves as one set of experimental scattering "data." Five more simulated experimental DCS's are shown in Fig. 1. These were generated by the pointwise introduction of random data scatter about the first DCS, using a normal distribution with the error bars of Paper II representing the standard deviation at each scattering angle, as shown in the figure.

Submitting each of these sets of generated DCS's to the data reduction procedures outlined in the previous section yields six different sets of potential parameters for each potential form. The sensitivity of the data to the potential at a given point is reflected by the potential energy spread among the six potentials at that point. Note, however, that this will not apply to potentials with shapes that are too rigid to reproduce the scattering results, because their parameters necessarily reflect only the gross scattering features.

For each function form used (excluding the MSV and $\mathrm{M}^{2} \mathrm{SV}$ forms which are treated independently in Sec. IV.A), each of the DCS curves is fit independently to potentials differing only in the values of their parameters. In Table I we show the corresponding average $r_{m}$ and $\epsilon$ parameters as well as the standard deviations of their means. Because the DCS data are sensitive to the well region, comparing the fitted $r_{m}$ and $\epsilon$ values with those of the original MSV potential is a first indication of the accuracy with which they are obtained. To this extent, their precision for a particular form is then reflected by the standard deviations of Table I. We also show in that table two goodness-of-fit statistical indices. We define the first as

$$
\chi^{2}=\sum_{i=1}^{n} g_{i}\left(I_{i}-\alpha \sigma_{i}\right)^{2} .
$$

At each of the $n$ scattering angles, the calculated cross section $\sigma_{i}$ must be scaled to the "experimental" DCS $I_{i}$ by the scaling factor $\alpha$ because only relative intensity measurements are made. The weighting factors (the $g_{i}$ ) are calculated from error bars corresponding to the data. Because the scaling factor is adjusted to bring the calculated and experimental DCS into vertical register, we may also calculate a confidence level for it,

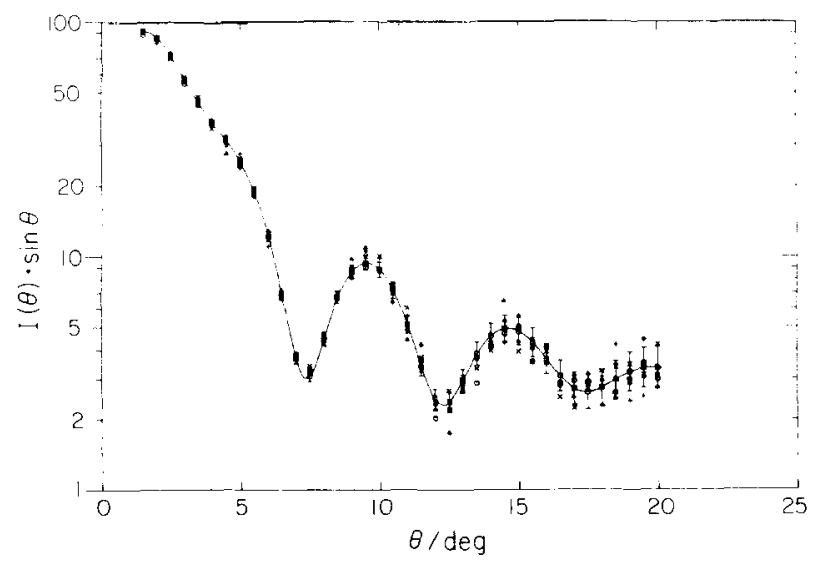

FIG. 1. He + Ar calculated DCS using the MSV potential. $I(\theta)$ is the DCS in arbitrary units at the scattering angle $\theta$, corresponding to out-of-plane detection of He atoms. The data points exactly on the DCS curve have error bars representing the assumed "experimental" noise at each angle. Other data points are chosen at random from a normal distribution using these error bars at each angle as one standard deviation. 
TABLE I. Results for the fits to the DCS having "experimental" data scatter, using an assumed MSV potential.

\begin{tabular}{|c|c|c|c|c|c|c|c|}
\hline Potential type & $\bar{r}_{m}(\AA)^{\mathrm{b}}$ & $\epsilon(\mathrm{meV})^{b}$ & $\sigma\left(r_{m}\right)(\AA)^{\mathfrak{C}}$ & $\sigma(\epsilon)(\mathrm{meV})^{c}$ & $\chi^{2} \mathbf{b}, \mathrm{d}, \mathbf{e}$ & $\frac{\Delta \bar{\alpha}_{0,95}, \mathrm{~b}, \mathrm{e}}{\alpha} \times 100 \%$ & $\frac{\Delta \alpha_{0.95}{ }^{p}}{\alpha} \times 100 \%$ \\
\hline Original MSV & 3.540 & 2.085 & $\cdots$ & $\cdots$ & $\cdots$ & $\cdots$ & $\cdots$ \\
\hline LJ $12-6$ & 3.441 & 2.030 & 0.007 & 0.044 & 916. (2) & 2.61 & 2.13 \\
\hline LaJ $n-6$ & 3.461 & 1.933 & 0.011 & 0.107 & $886 .(3)$ & 2.60 & 2.03 \\
\hline Exp-6 & 3.502 & 1.972 & 0.008 & 0.078 & $580 .(3)$ & 2.10 & 1.17 \\
\hline Kihara $n-6$ & 3.452 & 2.043 & 0.008 & 0.125 & $795 .(4)$ & 2.50 & 1.97 \\
\hline Klein-Hanley & 3.498 & 2.068 & 0.006 & 0.091 & $500 .(4)$ & 1.97 & 1.26 \\
\hline $\mathrm{BC}$ & 3.547 & 1.982 & 0.012 & 0.093 & $409 .(4)$ & 1.76 & 0.75 \\
\hline $\begin{array}{l}\text { BBP, second-order, } \\
\text { fit } c_{6} \text {, fix } c_{8}=0\end{array}$ & 3.533 & 2.133 & 0.005 & 0.046 & $554 .(5)$ & 1.90 & 0.49 \\
\hline $\begin{array}{l}\text { BBP, second-order, } \\
\text { fit } c_{5} \text { and } c_{8}\end{array}$ & 3.528 & 2.153 & 0.009 & 0.095 & $389 .(6)$ & 1.80 & 0.23 \\
\hline D-LJ, fit $m$ & 3.607 & 2.272 & 0.012 & 0.128 & $433 .(5)$ & 1.87 & 0.40 \\
\hline D-LJ, fix $m=6$ & 3.600 & 2.249 & 0.014 & 0.137 & 435. (4) & 1.84 & 0.42 \\
\hline SPFD, second-order & 3.537 & 2.189 & 0.009 & 0.089 & $408 .(4)$ & 1.79 & 0.20 \\
\hline SPFD, third-order & 3.523 & 2.084 & 0.006 & 0.114 & $364 .(5)$ & 1.71 & 0.15 \\
\hline SPFD, fourth-order & 3.533 & 2.071 & 0.023 & 0.121 & $358 .(6)$ & 1.72 & 0.14 \\
\hline
\end{tabular}

"This potential was chosen to correspond closely with one recently proposed for He-Ar. ${ }^{13}$ The MSV shape parameters are: $c_{6}=1.434, c_{8}=0.220, \rho_{2}=1.40, \beta=5.45$ of Eq. (7).

${ }^{b}$ Average of the values of the five fits.

'Standard deviation of the mean for the five fits.

Scattering intensity normalized to 500.0 at $5.0^{\circ}$; the number of parameters varied is given in parentheses.

${ }^{\circ}$ The average $\chi^{2}$ and $\Delta \alpha_{0,95} / \alpha$ statistical indices for the original MSV potential are $448(0)$ and $1.77 \%$, respectively.

${ }^{1}$ This $\Delta \alpha_{0.95} / \alpha$ statistical index is for fits to the DCS without data scatter.

$$
\Delta \alpha_{0.95}=t_{0.05}(n-k)\left\{\chi^{2} /\left[(n-k) \sum_{i=1}^{n} g_{i} \sigma_{i}^{2}\right]\right\}^{1 / 2} .
$$

Here $t_{0.05}(n-k)$ is Student's $t$-distribution statistic for a confidence level of 0.95 with $n$ data points and $k$ parameters. ${ }^{43}$ The goodness-of-fit statistic, $\Delta \alpha_{0.95} / \alpha$, is then independent of the arbitrary units chosen for the data, whereas $\chi^{2}$ is not. The former also takes into account that a fit with a greater number of parameters must have a lower $\chi^{2}$ in order to be of the same quality because $t_{0.05}(n-k)$ increases with $k$ for a given $n$; the $\Delta \alpha_{0.95} / \alpha$ statistic therefore provides a more appropriate measure of the goodness-of-fit. The five DCS's with data scatter as shown in Fig. 1 have an average $\Delta \alpha_{0.95} / \alpha$ value of $1.8 \%$. We find that fits having $\Delta \alpha_{0.95} / \alpha$ values considerably higher than this (say, $2.2 \%$ or greater) are not satisfactory.

Referring to Table I, we see that the simple two- and three-parameter potentials are generally too inflexible to reproduce the scattering results (i.e., they result in high values of $\Delta \alpha_{0.85} / \alpha$ ), even for the DCS with no data scatter. Comparison of plots of the derived LJ 12-6 potentials with that of the "known" MSV one show that the former have repulsive walls which are too "hard" (a generally observed deficiency ${ }^{2 x, 13.32}$ ) and values of $r_{m}$ which are consistently too small. By using LJ $n-6$ fits, the repulsive wall is made more flexible and allows a better determination of this region, but the values of $r_{m}$ and $\epsilon$ obtained are still systematically low. The exp- 6 shape is better able to reproduce the scattering results and the repulsive wall of the potential, but the resulting values of $r_{m}$ and $\epsilon$ are only marginally improved. The LJ $n-6$ and exp- 6 potential forms of Eqs. (1) and (2), respectively, are examples of three-parameter models using a single analytical parameterization. Another example of such a parameterization, also based on an inverse-power dependence of the radial distance, is the " $n(\rho)-6$ " potential of Maitland and Smith. ${ }^{35 a}$ It utilizes a repulsive term exponent $n$ which is a function of the radial distance [see Eq. (1) with $\gamma=0$ ], and has been of some use in fitting gas phase bulk properties. ${ }^{14 a, 35 a}$ As noted by Maitland and Smith, however, it cannot reproduce the multipole expansion appropriate for long-range interactions, nor can the shape parameter controlling the radial dependence of $n$ be related easily to physically meaningful features of the potential. ${ }^{35 a}$ As with the other potentials considered explicitly above, a more serious objection to the $n(\rho)-6$ parameterization is that it necessarily couples the long-range attractive region to the short-range repulsive region.

Only the well depth parameter is improved by the $\mathrm{Ki}$ hara modification of the LJ $n-6$ form. The DCS fits are marginally improved, but the repulsive region remains unrealistically "hard." Moreover, a very high statistical correlation ${ }^{43}$ exists between the repulsive parameters $\gamma$ and $n$, indicating that only one of them is truly independent. Consequently, using a Kihara-modified LJ 12-6 potential [i.e., fixing $n=12$ but fitting $\gamma$ of Eq. (1)] results in no substantial change from the analytically simpler and asymptotically more appropriate LJ $n-6$ model. On the other hand, the Klein-Hanley modification [Eq. (3)] of the LJ model yields better fits to the DCS data, and $r_{m}$ and $\epsilon$ values systematically low by only $1 \%$ (Table I). Although the attractive well is thus accurately reproduced, the short-range region is still too 
repulsive and the region beyond the minimum is insufficiently attractive. There is again a very high parameter correlation, in this case between the repulsive exponent $n$ and the reduced dispersion coefficient $c_{6}$, indicating that the short- and long-range regions are not independent within the framework of this model. This correlation precludes any meaningful values from being obtained for the dispersion coefficients. The $\mathrm{BC}$ parameterization [Eq. (4)] also yields good fits to the DCS data, and potentials whose $r_{m}$ parameter values are in good agreement with that of the MSV reference potential. However, the $\epsilon$ values are systematically low by $\sim 5 \%$. In addition, the region beyond the minimum is insufficiently attractive, as reflected by fitted values of the $C_{\delta}$ dispersion constant that are systematically too low by a factor of 3. Fixing the long-range part of the $\mathrm{BC}$ potential for systems for which the dispersion coefficients are known would be inappropriate, since this would also fix the shapes of the short-range repulsive and attractive minimum regions of the potential. We see that the $\mathrm{BC}$ parameterization does not allow the long- and shortrange regions of the potential to be independent of each other, precluding meaningful potentials from being obtained for them.

The highly flexible BBP potential [Eq. (5)] is the most successful of the forms with a single analytical expression. The second-order parameterization with only the first term of the dispersion series included (the three adjustable shape parameters are $c_{0}, \alpha$, and $A_{2}$ ) yields good fits to the DCS. The potential obtained and the fitted $r_{m}$ and $\epsilon$ parameters also agree very well with those of the original MSV potential, as seen in Table I. The attractive region immediately beyond the potential well is slightly too weak, a defect that can be cured by the addition of a second dispersion term, which further improves the DCS fitting.

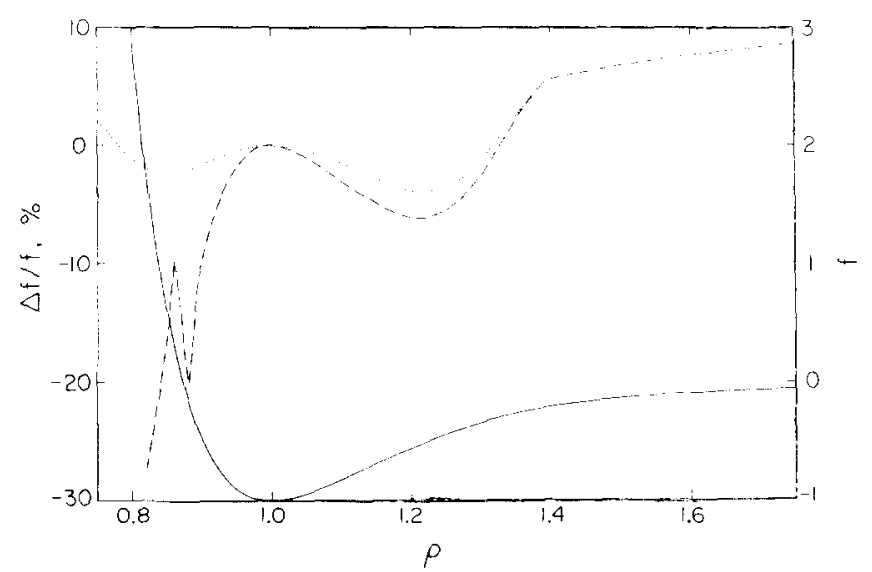

FIG. 2. Deviations of various reduced potentials from the original MSV form (broken curves). $(\Delta f / f) \times 100$ is the deviation, at each reduced interatomic separation $\rho$, of BBP forms obtained by direct fitting to the MSV form. The dashed curve shows the deviation of the second-order BBP form; the dotted line shows the deviation of the fourth-order BBP form. The solid curve, corresponding to the scale on the right, shows the original MSV reduced potential, $f$, for comparison. The discontinuity near $\rho=0.87$ is due to the zero of the potential; the absolute deviation, $\Delta f$, of the second-order BBP potential is 0.017 there.
In spite of the above advantages of the BBP parameterization, there are several difficulties which curtail its usefulness. By directly fitting the BBP form to other reduced potential functions, without the intermediate DCS calculations, we find that it is unable to reproduce the repulsive wall unless the expansion is carried out to fourth order $[N=4$ in Eq. (5): the fitted shape parameters are $c_{\hat{3}}, c_{8}, \alpha, A_{2}, A_{3}$, and $\left.A_{4}\right]$. As an example, we show in Fig. 2 the original MSV potential, and deviations from it for $N=2$ and $N=4$ BBP fits. It is apparent that although the fit is good in the attractive region, an excessive number of parameters is required to gain sufficient flexibility for the repulsive region. Because the nonreduced BBP potentials which are fit to the DCS's reproduce well the "correct" potential, as noted earlier, it is seen that the additional $r_{m}$ and $\epsilon$ parameters must be used to compensate for the reduced potential form's apparent lack of flexibility (at least for a reasonably low expansion order $N$ ); these potential well parameters thus cannot be determined independently of the potential shape, within the framework of the BBP model. A further objection to the BBP parameterization may be anticipated on theoretical grounds. The attempt made merely to "add on" the dispersion series to a suitable short-range potential is inappropriate at intermediate and short-range interatomic separations ${ }^{2 c, 29 a, 44}$ because the perturbation method used in the dispersion series formulation fails there. The fitted dispersion constants, as obtained from this model, cannot therefore be regarded as physically meaningful; this is reflected in the very high parameter correlations observed between any of the shape parameters of the second-order $(N=2)$ DCS fitting, even if $c_{8}$ is held fixed.

The piecewise analytical potentials may be expected to reduce the problem of correlations between the longand short-range components of the potential. For example, use of the double-LJ model [Eq. (6)] enables good fits to be obtained, with the long-range potential being recovered very well. The short-range repulsion is slightly too steep, but a more serious deficiency is in the well region, as is apparent from Table I. Fitted $r_{m}$ and $\epsilon$ values are systematically too large, by about $2 \%$ and $10 \%$, respectively. Thus it appears that the repulsive region parameters $\left(n_{1}\right.$ and $\left.m\right)$ are only weakly statistically correlated with the attractive region parameter $\left(n_{2}\right)$. The motivation for using the double-LJ parameterization is to decouple these two regions of the potential. However, $n_{1}$ and $m$ are still highly correlated with the well region parameters $r_{m}$ and $\epsilon$. Moreover, there exists a high parameter correlation among $n_{1}$ and $m$, leaving only one of them available to determine independently the shape of the repulsive wall. Indeed, fixing $m=6$ and fitting to the DCS with the remaining four parameters results in little change of either the DCS or of derived potentials (Table I).

The MSV form [Eq. (7)] could not be tested in the usual manner because of its use as the starting potential. This form was, as a result, tested independently against a Klein-Hanley reference potential, as discussed in Sec. IV.A. However, it is already clear that the MSV form also suffers from a parameterization that forces corre- 
lation between the repulsive region and the attractive well, since both regions are essentially determined by, and are very sensitive to, the Morse $\beta$ parameter. In order to avoid this restriction, an exponential repulsion may be splined onto the MSV curve in the short-range region, giving the ESMSV form. ${ }^{34 c}$ This modification has been used in a number of studies to obtain potentials simultaneously describing dilute gas transport and equation-of-state properties (which are primarily sensitive to the repulsive interactions) and the rmal energy scattering results. ${ }^{11,13}$ This approach, however, still leaves a substantial portion of the low-energy repulsive wall parametrically coupled to the attractive well. In order to avoid this coupling, we may use the $\mathrm{M}^{2} \mathrm{SV}$ potential with a different Morse parameter $\beta^{\prime}$ for interatomic separations $r<\sigma$. For either the MSV or $\mathrm{M}^{2} \mathrm{SV}$ potentials, however, changes in the long-range region are not restricted to values of $\rho$ beyond $\rho_{2}$ because of the effect of such changes on the splined portion between $\rho_{1}$ and $\rho_{2}$. ${ }^{14 a}$

A similar problem could also be expected to plague the SPFD potentials [Eq. (8)]. In this case, changes in the long-range region also affect the potential for all $\rho$ $\leqslant \rho_{f}$ because of the smoothness conditions imposed upon the two highest-order coefficients. Fortunately, as we show in Sec. IV, the fitted SPFD potentials (with only the $c_{6}$ term beyond $\rho_{f}=1.6$ and $N=2,3$, or 4 ) can have their long-range regions modified with no substantial effect on the potential for $\rho \gtrless 1$. 3. Consequently, these forms should allow good recovery of the potential in the long-range region. Furthermore, they all show sufficient flexibility to fit the scattering data just as well as the original MSV potential does. This is reflected in the good agreement between the original and fitted potentials throughout the well (Table I) and adjacent regions, although the repulsive region seems to be slightiy too steep, as seen in Fig. 3. Values of $r_{m}$ and $\epsilon$ are recovered to within $0.5 \%$ and $5 \%$, respectively. Most of the discrepancy in $\epsilon$ is due to the second-order SPFD fits $[N=2$ in Eq. (8)], which have systematically deeper wells than those of higher order; third- and fourth-order fits each reproduce $\epsilon$ to within $1 \%$. These results substantiate previous claims that the SPFD potential form is eminently suitable for use in analyzing thermal scattering results. ${ }^{15,18,38 b}$ It should be emphasized that the second-order potentials are obtained with only two of the available shape parameters $\left[c_{6}\right.$ and $b_{0}$ of Eq. (8) $]$. The third-order potentials show additional flexibility and result in better fits to the DCS, but those of fourth order show no further improvement.

\section{UNIQUENESS, FLEXIBILITY, AND SENSITIVITY}

Because of the ability of the SPFD potential to adequately reproduce the scattering results and to recover the original MSV potential, we have investigated it further. We pay particular attention in this section to its uniqueness, flexibility, and sensitivity.

\section{A. Uniqueness}

Previous users of this potential cautioned that there may exist multiple local minima on the least-squares hypersurface; that is, the fitted potential may depend on the first-guess parameters of the iterative fitting scheme. ${ }^{15}$ We address this problem by arbitrarily beginning second-order SPFD fits with parameters corresponding to the LJ 12-6 shape; $b_{0}=36.0$ from the curvature at the bottom of the well, and $c_{\hat{0}}=2.0$ from the longrange behavior. Starting values of $r_{m}$ and $\epsilon$ were up to $10 \%$ and $30 \%$ different from their "correct" values, respectively. Fitting to one of the DCS with data scatter gives the same parameter values, within four to six iterations, as those obtained with first-guess parameters corresponding to the original MSV potential. ${ }^{45}$

Initial values of $r_{m}$ and $\epsilon$ cannot be too different from the "correct" ones, however. A difference of $20 \%$ and $50 \%$, respectively, in these parameters is sufficient to cause the iterative scheme to converge to a grossly incorrect potential. In this case, the difficulty is indicated by the very poor fit and contorted potential obtained and may easily be avoided $a$ priori. For example, initial fitting with any one of the simpler functional forms may be used to obtain values of $r_{m}$ and $\epsilon$ within the (apparent) convergence radius of the second-order SPFD potential. Indeed, the range of values obtained for the $r_{m}$ and $\epsilon$ parameters from all the fits to each of the DCS with data scatter are within $3 \%$ and $15 \%$ of their "correct" values, respectively.

Using the smoothness conditions, the second-order fit provides a reliable value for the $b_{1}$ parameter which can be used as a starting point for the third-order fit. This in turn provides a good guess for the $b_{2}$ parameter; SPFD potentials with unique parameter values can thus be obtained for fits up to at least fourth order.

A possible bias in our analysis is that the SPFD potential might be adaptable only to the MSV shape, in part

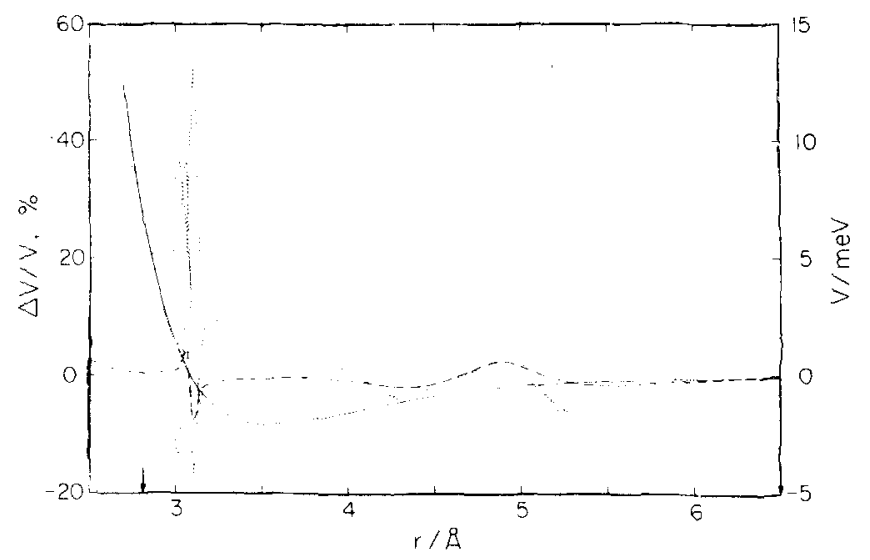

FIG. 3. Deviations of various potentials from the original MSV one (broken curves). $(\Delta V / V) \times 100$ is the deviation, at each interatomic separation $r$, of third-order SPFD potentials obtained by fitting to the "experimental" DCS's. The dashed curve shows the deviation of the SPFD form fit to the DCS having no data scatter; the dotted curves show the deviations for fits to three of the DCS's with data scatter. The solid curve, corresponding to the scale on the right, shows the original MSV potential, $V$, for comparison. The discontinuities near $r=\sigma$ $=3.1 \AA$ are due to the zero of the potential; the absolute deviations, $\Delta V$, for the dotted potentials are about $\pm 0.2 \mathrm{meV}$ there. The arrows at $r=2.8 \AA$ and $r=6.5 \AA$ delineate the range of sensitivity of the "experimental" DCS to the potential (see Sec. IV, B of the text). 
TABLE II. Results for the fits to the DCS having "experimental" data scatter, using an assumed Klein-Hanley potential. $a_{4} b$

\begin{tabular}{llllllll}
\hline \hline Potential Type & $\bar{r}_{m} / \AA$ & $\bar{\epsilon} / \mathrm{meV}$ & $\sigma\left(r_{m}\right) / \AA$ & $\sigma(\epsilon) / \mathrm{meV}$ & $\bar{\chi}^{2}$ & $\left(\overline{\left.\Delta \alpha_{0.95} / \alpha\right) \times 100 \%}\right.$ & $\left(\Delta \alpha_{0.95} / \alpha\right) \times 100 \%$ \\
\hline Original Klein-Hanley & 3.500 & 2.500 & $\ldots$ & $\ldots$ & $\ldots$ & $\ldots$ & $\ldots$ \\
MSV & 3.496 & 2.783 & 0.011 & 0.098 & $324 .(4)$ & 1.93 & 0.59 \\
M 2 SV & 3.516 & 2.481 & 0.016 & 0.172 & $253 .(5)$ & 1.74 & 0.22 \\
SPFD, third-order & 3.469 & 2.603 & 0.009 & 0.131 & $244.5)$ & 1.70 & 0.17 \\
\hline
\end{tabular}

${ }^{2}$ This potential was chosen to correspond closely with one recently proposed for He-Ar. ${ }^{14}$ The Klein-Hanley

[Eq. (3)] shape parameters chosen are: $c_{6}=1.80 ; n=12.0$, and therefore $c_{8}=0.30$.

${ }^{b}$ Headings have the same meanings as in Table $I$.

because of their similar piecewise long-range behaviors and smoothness conditions. To test this possible bias, we calculated a new input "experimental" DCS from an assumed potential of the Klein-Hanley form [Eq. (3)], rather than of the MSV shape. The former is represented by a single analytical function bearing no mathematical relation to any but the $\mathrm{LJ}$ family of potentials [Eqs. (1) and (6)], and includes a second dispersion term in $\rho^{-8}$. We then fit this DCS, including "data scatter", to the third-order SPFD form (fitting the $r_{m}, \epsilon, c_{\bar{b}}, b_{0}$, and $b_{1}$ parameters, but setting $c_{8}=0$ ). To allow a more rigorous evaluation of the MSV and $M^{2} S V$ forms than that outlined towards the end of the previous section, we also submit the "experimental" DCS's to fits with these potentials (also without terms in $\rho^{-8}$ ). The results are displayed in Table II and Fig. 4.

From Table II, it is seen that the "correct" range parameter $r_{m}$ is recovered quite well by all three potentials (error $<1 \%$ ). The third-order SPFD potential is able to reproduce the "experimental" DCS's calculated from the Klein-Hanley assumed potential just as well as

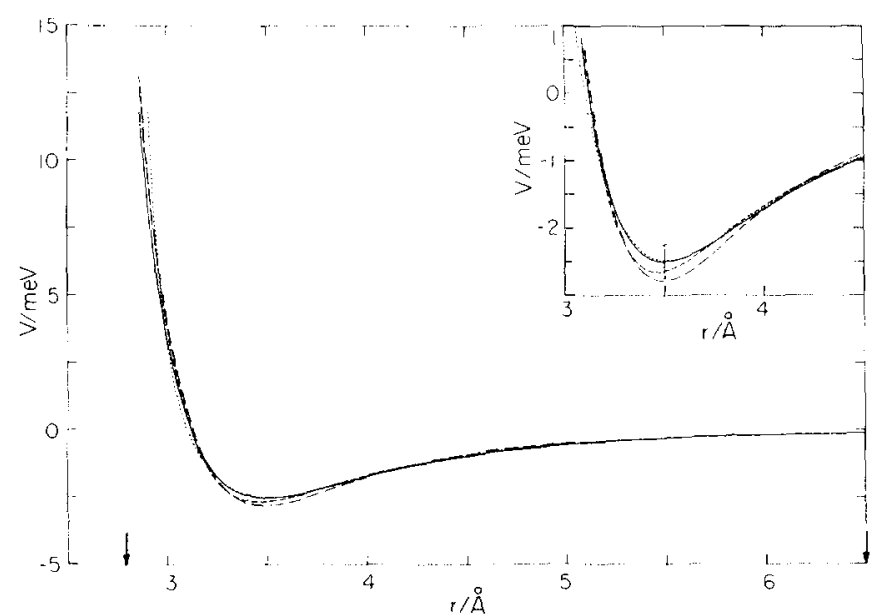

FIG. 4. Potentials obtained from fits to the "experimental" DCS's generated using the Klein-Hanley potential. $V$ is the interatomic potential at each interatomic separation $r$; the inset magnifies the vertical scale of the potential energy minimum region by a factor of 2.5. The solid curve is the original KleinHanley potential and the broken curves show the average potential $V$ at each $r$ for a particular potential form. Long dashed curve: MSV potential; dotted curve: $M^{2} S V$ potential; short dashed curve: third-order SPFD potential. The error bar in the inset indicates the estimated uncertainty of $\pm 10 \%$ at the potential minimum; the arrows on the abscissa are placed as in Fig. 3, those calculated from the MSV assumed potential (Table I); although the average well depth parameter value $\epsilon$ is too large by about $4 \%$, this is within the estimated error limit of $10 \%$ for the attractive well region of the potential (see the end of this section). For the MSV potential, the average parameter values and quality-of-fit statistical indices are less favorable than for the third-order SPFD potential; its $\epsilon$ value is about $11 \%$ too large and the fitting quality is not quite as good. Use of the $\mathrm{M}^{2} \mathrm{SV}$ potential, which has a second Morse function to describe repulsive interactions independently of the attractive well, results in potentials which have very good average parameter values $\left(r_{m}\right.$ and $\epsilon$ values in error by $\left.<1 \%\right)$. The fits are of the same quality as those of the thirdorder SPFD potentials, although the short-range repulsive behavior of the $\mathrm{M}^{2} \mathrm{SV}$ potential is too "hard" (by $>40 \%$ at $r=2.9 \AA$ compared with $<15 \%$ for the SPFD and MSV potentials).

The second series of simulation results, using a Klein-Hanley "reference" potential, thus clearly shows that the SPFD potential is sufficiently flexible [with $N \geqslant 3$ in $\mathrm{Eq}$. (8)] to recover two realistic potentials of very distinct mathematical forms, both of which are quite different from the SPFD form. This second simulation study complements the first, which in using an MSV "reference" potential, showed that of all the mathematical forms analyzed [Eqs. (1) through (8), except for (7)], only the SPFD one was adequate to reproduce the simulated DCS's and to recover the original potential. Our analysis of the MSV and $\mathrm{M}^{2} \mathrm{SV}$ potentials [Eq. (7)] in the second simulation study clearly indicates that the $\mathrm{M}^{2} \mathrm{SV}$ potential may be preferred over the MSV potential, and perhaps even over the SPFD potential in the attractive well region. It is important to note that the third-order

TABLE III. Dependence of the third-order SPFD fitted dispersion coefficients on the joining point $\rho_{f}$.

\begin{tabular}{lccc}
\hline \hline$\rho_{f}$ & $\bar{C}_{6}\left(\mathrm{eV} \AA^{6}\right)^{\mathrm{a}}$ & {$\left[\left(\bar{C}_{6}-C_{6}\right) / C_{6}\right] \times 100 \%^{\mathrm{b}}$} & {$\left[\sigma\left(C_{6}\right) / C_{6}\right] \times 100 \%^{\mathrm{c}}$} \\
\hline 1.4 & 5.84 & -0.8 & 5.8 \\
1.6 & 5.64 & -4.2 & 4.9 \\
1.8 & 5.51 & -6.5 & 6.5 \\
2.0 & 5.74 & -2.5 & 14.8 \\
2.2 & 6.30 & +7.0 & 26.7 \\
\hline
\end{tabular}

${ }^{a} \bar{C}_{6}$ is the average of the dispersion coefficients obtained from the fits to the five "experimental" DCS's with data scatter.

${ }^{b} C_{6}=5.89 \mathrm{eV} \AA^{628 a}$ is the dispersion constant used in the original MSV potential.

${ }^{c} \sigma\left(C_{6}\right)$ is the standard deviation of the fitted coefficients. 
TABLE IV. Definition of regions for the numerically modified MSV potential

\begin{tabular}{lll}
\hline Region & Range $^{2}$ & Sensitivity $^{\mathrm{b}}$ \\
\hline 1. Repulsive wall & $\rho<0.87$ & $\pm 30 \%$ \\
2. Attractive well & $0.87<\rho<1.40$ & $\pm 10 \%$ \\
3. Attractive tail & $\rho>1.4$ & $\pm 20 \%$ \\
\hline
\end{tabular}

${ }^{a}$ Early attempts to define four regions split region 2 into two parts at $\rho=1.10$; the attractive tail region was then defined by $\rho>1.5$.

Minimum change in the potential shape needed to produce statistically significant changes in the corresponding DCS. ${ }^{54}$

SPFD and the $M^{2} S V$ potentials, both of which have five adjustable parameters, are indistinguishable from each other and from the Klein-Hanley reference potential (see Fig. 4) within the estimated uncertainty of the derived potential energy curves (see the end of this section). The MSV potential, having only four parameters, is distinguishable from the reference potential (Fig. 4), indicating that it provides a representation for the potential which, in view of the improvements to be gained by using the five-parameter models, is not quite as adequate. Although the $\mathrm{M}^{2} \mathrm{SV}$ potential appears to be slightly superior to the third-order SPFD potential, we feel that the latter provides a more unified representation ${ }^{15,18}$ as well as having a sounder basis in theory ${ }^{17}$; we also recall that the $\mathrm{M}^{2} \mathrm{SV}$ potential is too "hard" in the short-range region $(r<2.9 \AA)$, despite the sensitivity of the DCS to the potential there as shown later in this section.

We also assess the degree to which independent scattering experiments may complementarily be used to determine the interaction potential. Using the five sets of third-order SPFD parameters obtained by fitting the individual DCS's with "experimental data scatter", we calculate the corresponding five potentials and display the arithmetic average of these at each interatomic separation in Fig. 5. It can be seen that the data obtained from the five "independent experiments" are indeed complementary; the averaged potential is significantly closer to the original MSV potential than are any of the thirdorder SPFD fits to the DCS (compare with Fig. 3), with the only significant discrepancy occurring in the repulsive wall region.

\section{B. Flexibility and sensitivity}

Besides fitting the SPFD potential to the "experimental" DCS's, we may also fit its reduced form to other reduced potential functions, without the intermediate DCS calculations, as was done with the BBP potential in Sec. III. The reduced potentials of Eqs. (1) through (7) with "reasonable" shape parameter values are reproduced almost identically by the numerically fitted SPFD shapes (third-order or higher), demonstrating the latter's ability to fit interaction potentials of any reasonable form in the region beyond about $\rho=0.65(\lambda=-0.5)$. Thus, when fitting to the DCS's, the two reduction parameters $r_{m}$ and $\epsilon$ have a large degree of independence from the shape parameters (in contrast, for example, to the BBP potential, see Sec. III); the resulting potential energy function in general, and the well depth and position, in particular, are parameterization independent.

With the flexibility attained by the SPFD potentials, we now embark on an investigation of the possibility of analytically varying only a selected region of the potential. This would enable a determination to be made of the sensitivity with which each region is probed by the thermal scattering results.

Classically, of course, this could be determined simply by reference to the deflection function, ${ }^{31,47}$ which would also serve to locate the limits of the interatomic distance range sampled by the experiment (hereafter called the range of sensitivity). Such arguments have been used in some instances to provide an indication of the correspondence between specific regions of the potential and the scattering results. On this classical basis, it has been claimed that these experiments are insensitive to the long-range region, ${ }^{14,46}$ or even that they are sensitive only to the low-energy repulsive wall of the potential. . $^{20,49}$ The further implication is that the attractive well and long-range behavior are probed only by very low scattering angles, since the classical rainbow occurs at a laboratory angle of $3.7^{\circ}$ for this system. However, the quantum mechanical results discussed below indicate a greater range of sensitivity.

The SPFD potential form is suitable for a determination of the upper limit of the range of sensitivity because of the availability of the $\rho_{f}$ parameter. Thus, $C_{6}$ coefficients $^{38}$ obtained by fitting the various DCS having data scatter may show large deviations from the "correct" value and from each other, only for cases in which $\rho_{f}$ lies beyond the range of sensitivity of the potential to the scattering results. For third-order fits, the average of the $C_{6}$ values obtained (see Table III) agree well with the $C_{6}$ coefficient of the original MSV potential for

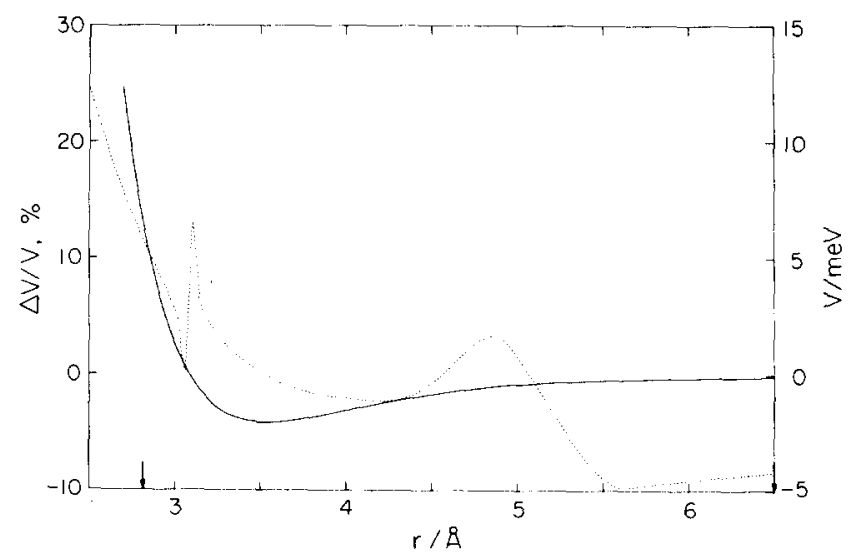

FIG. 5. The deviation of a numerically averaged potential from the original MSV one (broken curves). $(\Delta V / V) \times 100$ is the deviation, at each interatomic separation $r$, of the numerical average of the five third-order SPFD potentials of Fig. 3. The solid curve, corresponding to the scale on the right, shows the original MSV potential, $V$, for comparison. The discontinuity near $r=3.1 \AA$ is due to the zero of the potential; the absolute deviation, $\Delta V$, of the numerical potential is $-0.02 \mathrm{meV}$ there. The arrows on the abscissa are placed as in Fig. 3. 
all the $\rho_{f}$ 's tested $\left(\rho_{f}=1.4,1.6,1.8,2.0\right.$, and 2.2). However, standard deviations of those $C_{6}$ values for each $\rho_{f}$ are below $10 \%$ only for the lower values of $\rho_{f}(1.4$, 1.6, and 1.8). The much less consistent $C_{6}$ values for $\rho_{f}=2.0$ and 2.2 indicate that the data are not sensitive to the potential beyond about $\rho=1.8$. To assess the extent to which the scattering results are sensitive to the long-range region, we may also fit the $c_{8}$ parameter of the SPFD potential, for $\rho_{f}=1.6$. Third-order fits to the DCS with data scatter yield widely varying values of $C_{8}$. Furthermore, through statistical correlations with the $c_{8}$ parameter, even the fitted values of $C_{6}$ deteriorate markedly.

Thus the $C_{6}$ coefficient obtained from the third-order SPFD fits should be reliable to within $15 \%$, insofar as the contribution from higher-order dispersion terms is small (for the original MSV potential, $c_{8} \rho^{-8} / c_{6} \rho^{-6}=6 \%$ at $\rho_{f}=1.6^{13}$ ); however, these higher-order terms may not be determined reliably ${ }^{44,50}$ from experiments at present. It is seen that the upper limit of the range of sensitivity of about $6.5 \AA(\rho=1.8)$ extends well beyond the $4.8 \AA$ value imposed by classical considerations.

The approach we use to determine the lower limit of sensitivity is to modify exponentially the third-order fitted SPFD potential at chosen values of $\rho_{i}$, as discussed in Sec. II. The DCS's calculated with these potentials begin to show manifestations of the modification (which "hardens" the repulsive wall) for values of $\rho_{i}$ greater than about 0.82 . It is interesting to note that the first effect of increasing $\rho_{i}$ is to decrease the DCS at angles beyond the first minimum ( $10^{\circ}$ in the lab frame). Values of $\rho_{i}$ nearer the zero of the potential (at $\rho=0.873$ ) cause radical changes throughout the angular range of the calculated DCS. In particular, the low-angle shoulder near the classical rainbow becomes smoother. The $\rho_{i}$ obtained from this analysis corresponds to significant differences between the exponentially modified and original SPFD potentials for values of $\rho$ below about 0.81 . Thus the lower limit of sensitivity to the potential is about $2.8 \AA$, very close to the $2.9 \AA$ value determined from a classical analysis. Because of the highly repulsive wall encountered in short-range interactions, it is not surprising that the lower limit of sensitivity would be estimated more reliably by classical arguments than the long-range interactions.

Having thus determined an approximate range of the potential sampled by thermal DCS scattering results for this system, we now address the question of the accuracy with which the potential is determined. The original MSV potential is numerically modified within each of a few chosen regions having the somewhat arbitrary limits imposed in Table IV. The SPFD shape obtained by direct fitting to the new reduced potential may then be analyzed for its ability to adjust to these discrete changes.

With four available shape parameters $\left(b_{0}, b_{1}, b_{2}\right.$, and $c_{6}$ ) of the fourth-order SPFD potential, an initial attempt to specify a like number of regions was made. As indicated toward the end of Sec. III, the long-range region of the potential is largely independent of the others; the short-range region apparently also shows such indepen- dence. However, within the SPFD parameterization, this initial attempt indicates that the intermediate range should be treated as one, rather than as two independent regions. Subsequent fitting to the MSV potential with numerical modifications in three regions instead of four show a moderate degree of success in decoupling the long- and short-range behaviors from the intermediate region, as well as from each other. It may also be seen from Fig. 6 that the ability of the fourth-order SPFD model to fit the modified MSV potential is largely independent of the magnitude of the changes made.

It is also apparent that because the reduced SPFD potential can adjust to changes in just three regions, there are only three uncorrelated shape parameters within the range of interatomic separations to which the DCS data are sensitive. This conclusion is further supported by the earlier observation that improvements in the DCS fits are noted for SPFD potentials up to third order, but not for higher orders. In addition, since the $r_{m}$ and $\epsilon$
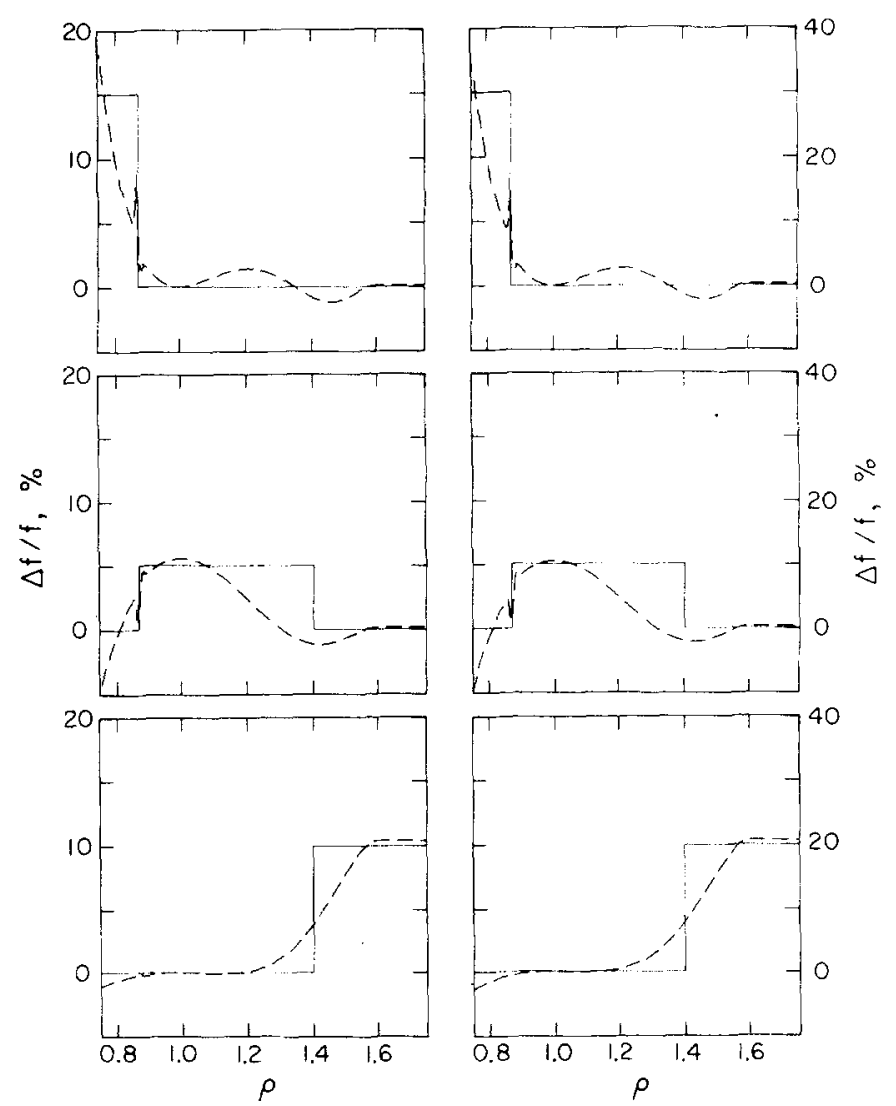

FIG. 6. Deviations of various reduced potentials from the original MSV potential form (broken curves). $(\Delta f / f) \times 100$ is the deviation, at each reduced interatomic separation $\rho$, of fourthorder SPFD potential forms obtained by direct fitting to numerically modified MSV potentials. Solid lines are the step-function modifications made to the original MSV potential; dashed lines show the response of the SPFD potential forms in fitting them. Graphs in the right column represent changes twice as large as the ones in the left column (note scale change). The upper panels show the response to $15 \%$ and $30 \%$ changes in the repulsive wall region; the middle panels, to $5 \%$ and $10 \%$ changes in the attractive well region; and the lower panels, to $10 \%$ and $20 \%$ changes in the attractive tail region of the potential. The discontinuity near $\rho=0.87$ is due to the zero of the potential. 


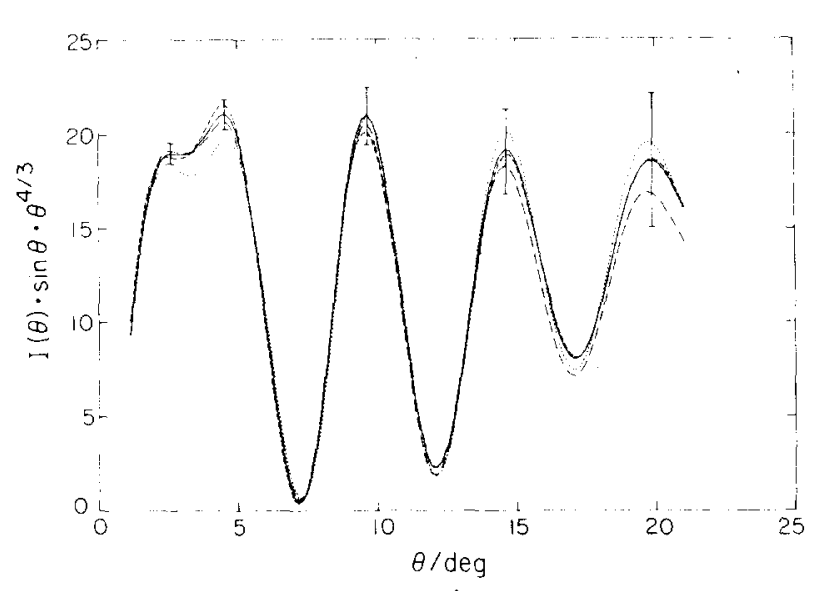

FIG. 7. He + Ar calculated DCS's at a collision energy of $64 \mathrm{meV}$ using the fourth-order SPFD potentials fitted to the numerically modified MSV potentials. $I(\theta)$ is the DCS in arbitrary units at the scattering angle $\theta$, corresponding to out-ofplane detection of He atoms. The solid line corresponds to scattering calculated from the original MSV potential, with error bars representing the assumed "experimental" noise at representative angles. The long dashed line corresponds to a potential modification of $+30 \%$ in the repulsive wall region; the dotted line, to a $+10 \%$ modification in the attractive well region; and the short dashed line, to a $+20 \%$ modification in the attractive tail region. Note that the ordinate is very sensitive to the oscillatory structure of the DCS. ${ }^{3 !}$

parameters independently determine the scale of the potential, we conclude that up to five independent parameters may justifiably be used for fitting DCS data of the type being considered, with SPFD potentials.

The effects on the calculated DCS due to these parametric modifications of the potential may most easily be seen by neglecting the loss of angular resolution caused by experimental averagings (see Fig. 7). Alterations in the intermediate range produce the largest effects, especially in the rainbow region around $3.7^{\circ}$ where an increased attraction produces a more pronounced shoulder. Similar changes in the long-range attraction are reflected primarily in an increased cross section in the low-angle portion of the DCS. The entire low-angle region (below $10^{\circ}$ ) is largely unaffected even by the more drastic short-range modifications made. The effects of the latter appear mostly in the wider-angle oscillatory pattern of the DCS (beyond $15^{\circ}$ ).

By repeating the type of calculations displayed in Fig. 7 at some energy different from $64 \mathrm{meV}$ (see end of Sec. II), the sensitivity of the DCS to different regions of the potential may crudely be gauged as a function of the collision energy. Such calculations are necessitated by the nonmonotonic relation between the collision energy and the sensitivity with which the potential may be determined, in a given range, from arbitrarily normalized DCS experiments. For example, at very high collision energy, attractive interactions are insignificant compared with repulsive ones, while at very low collision energy, the DCS becomes isotropic, and information can be extracted only if absolute measurements are made. ${ }^{51}$ Figure 8 shows the effect on the DCS of making the parametric modifications to the MSV reference po- tential shown on the right-hand panels of Fig. 6 , but at a collision energy of $19 \mathrm{meV} .{ }^{52}$ Thus Fig. 8 is analogous to Fig. 7, but at only $30 \%$ of the collision energy. We see again that large changes in the repulsive wall region of the potential affect the DCS only for wide scattering angles (beyond $\sim 30^{\circ}$ ), but that these effects are less pronounced than at the higher energy. The low-energy DCS is thus less sensitive to the weakly repulsive wall than is the high-energy one. Comparisons between Figs. 7 and 8 regarding the effects of potential modifications in the attractive well and attractive tail regions show that the long-range potential is more sensitively probed by the low-energy DCS, but that the attractive well region is not (cf. the first three oscillations). The overall effect of lowering the collision energy from $\sim 64$ to $\sim 19 \mathrm{meV}$ is evidently to shift the range of interatomic separations probed most sensitively by the DCS experiments, from the attractive minimum region outward to the attractive tail region. That is, the DCS becomes dominated by the long-range attractive potential. Consequently, parametric forms most suitable for the analysis of low-energy DCS data should have more flexibility in the attractive tail region than those used for extracting the potential from higher-energy DCS curves. This may easily be accomplished by introducing a second adjustable dispersion term into the MSV, $\mathrm{M}^{2} \mathrm{SV}$ or SPFD potentials [Eqs. (7) and (8)]. Flexibility of the repulsive wall independent of the attractive well is probably no longer needed at the lower energy; the MSV or second-order SPFD potential forms are probably sufficient.

Although the above conclusions ${ }^{13.53}$ appear to be similar to ones based on classical arguments, the differences must be emphasized. Changes in the attractive region of the potential produce significant differences throughout the DCS, whereas such differences classically would be less pronounced at angles beyond the rainbow. Furthermore, these potential changes (at a given energy) affect the amplitudes of the wide-angle oscillations (but not their positions), which are especially intensified by a stronger attractive region. These conclusions are,

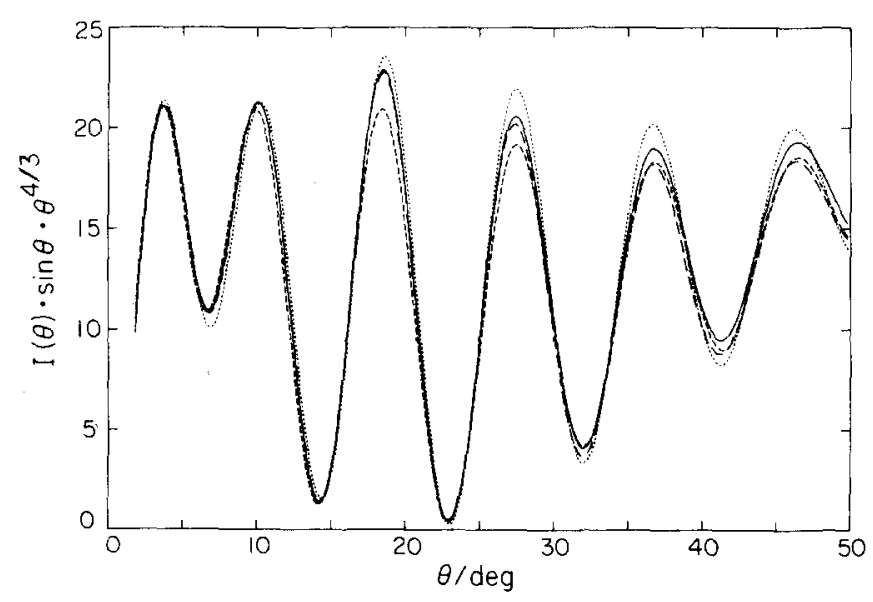

FIG. 8. He+Ar calculated DCS's at a collision energy of 19 meV, using the fourth-order SPFD potentials fitted to the numerically modofied MSV potentials. Symbols are as in Fig. 7; error bars from that figure are transferrable to Fig. 8 provided the signal-to-noise ratio is the same for both energies. Note the difference in the angle scales of these two figures. 
of course, somewhat predicated upon the experimental conditions, e.g., collision energy, resolution, signalto- noise ratios, etc.

The above trends are largely preserved upon inclusion of the simulated experimental ave raging. The effects are, of course, less visible but are nevertheless statistically appreciable. ${ }^{54}$ Significant deteriorations in the DCS fitting at $64 \mathrm{meV}$ appear to the extent shown in the last column of Table IV, indicating that the accuracy with which the potential can be determined in the repulsive wall, attractive well, and attractive tail regions defined in that table are approximately $30 \%, 10 \%$, and $20 \%$, respectively. Approximately the same sensitivites are indicated by the range of third-order SPFD potentials fitted to the five DCS's with data scatter (Fig. 3). Referring to the standard deviation columns of Tables I and II, it is apparent that for the realistic experimental conditions assumed in the present simulation (see the end of Sec. II), the DCS's are sensitive, for a particular potential shape, to changes of the $r_{m}$ and $\epsilon$ parameters of about $0.5 \%$ and $5 \%$, respectively. ${ }^{11}$ This reflects the precision with which these parameters are obtained from the fits to any one of the DCS's with data scatter. The accuracy of the $r_{m}$ and $\epsilon$ parameters approach this level of precision only for those functional forms having sufficient flexibility to parametrically decouple different regions of the potential from each other. This is best accomplished by the $\mathrm{M}^{2} \mathrm{SV}$ and third-order SPFD potential forms. Using them, the well depth parameter $\epsilon$ is accurate (i.e., agrees with the "known" $\epsilon$, as well as being precise) to within $\sim 10 \%$, while $r_{m}$ is similarly accurate to within $\sim 2 \%$ (Table II). The distance $\sigma$ at which the potential changes sign is completely determined by $r_{m}$ and the other potential parameters, and is accurate to within $\sim 1 \%$ (not shown in Table II, but see Fig. 4). Under the conditions considered in this paper, these are the limits to which the interatomic pair potential may be determined by thermal DCS scattering measurements.

\section{SUMMARY AND CONCLUSIONS}

In this paper we have attempted to extend the usefulness of the techniques prevailing for the determination of interatomic potentials. Our method, as applied to crossed molecular beam experiments for highly quantal systems, is to begin with a "known" spherically symmetric interaction. This serves as the basis for calculating scattering cross sections as the hypothetical "experimental" data, to which data scatter is added. By fitting these cross sections to interatomic potentials, which are functionally different from the original one, we have arrived at the following qualitative conclusions:

1. Different parameterizations for different regions of the potential are generally helpful in avoiding high statistical parameter correlations.

2. The mathematical form assumed to represent the potential energy functions must be chosen with great care if accurate results are to be obtained. This choice may be dependent upon the collision energy.

3. The thermal DCS scattering results are sensitive to the potential significantly beyond the limits imposed by classical mechanics.

These conclusions are substantiated quantitatively for collision energies $\sim 30$ times the van der Waals attractive well depth as follows:

1. The potential forms most suitable for accurately reproducing both the scattering patterns and the original potential have at least four parameters. Up to five independent potential parameters may justifiably be determined.

2. The SPFD and $M^{2} S V$ forms are the most success ful of the potentials in the above sense, combining a high degree of flexibility with uniqueness. We concur with the conclusion of Bickes and Bernstein ${ }^{15}$ that the SPFD form provides a unified and accurate parameterization for the attractive well and adjacent regions of the potential. The $\mathrm{M}^{2} \mathrm{SV}$ form, with the same number of parameters as the third-order SPFD form, is an equally successful representation of the potential energy, but it does not provide as systematic or as unified a representation compared with the SPFD form, nor is it quite as adequate in the short-range repulsive region.

3 . The sensitivity is greatest in the well region, allowing $r_{m}$ to be determined with an accuracy of $\pm 2 \%$ and the potential to $\pm 10 \%$, for realistic experimental conditions. These experiments are also very sensitive to the shape of the well region and, to lesser extents, to those of the long- and short-range regions.

4. Unique potentials may be obtained from suitable analyses (in the sense of the above conclusions) of scattering results of the type considered here, even under realistic experimental conditions. This is indicated by the indistinguishability (within error limits) of the SPFD and $M^{2} S V$ potentials from the original potential assumed in the simulation, despite the very different parameterizations of all three of these potentials.

Although the above conclusions are based on the poignant example of He-Ar thermal scattering, they can be extended to other highly quantal systems. These include the range of systems for which the dimensionless $A$ and $B$ parameters ${ }^{31}$ do not differ excessively from those explicitly considered in this paper $(A \simeq 20$ to 40 and $B \simeq 50$ ). Within the validity of the central-field assumption for atom-molecule systems, the conclusions enumerated here may be extended, allowing a more systematic approach to the problem of analyzing scattering data for nonspherical systems. ${ }^{20}$

\section{ACKNOWLEDGMENT}

We wish to thank Ambassador College for generous use of its computing facilities.

${ }^{1} J$. O. Hirschfelder, C. F. Curtiss, and R. B. Bird, Molecular Theory of Gases and Liquids (Wiley, New York, 1954).

${ }^{2}$ For recent reviews pertaining especially to molecular beam studies of intermolecular potentials, please see (a) U. Buck, Adv. Chem. Phys. 30, 313 (1975); (b) Rev. Mod. Phys. 46, 369 (1974); (c) G. C. Maitland and E. B. Smith, Chem. Soc. 
Rev. 2, 181 (1973); (d) J. P. Toennies, in Plizsical Chemistry: An Advanced Treatise, edited by H. Eyring, D. Henderson, and W. Jost (Academic, New York, 1974), Vol. 6A, p. 227.

${ }^{3}$ See also (a) A. A, Abrahamson, Phys, Rev, 178, 76 (1969); (b) Ch. Schlier, Annu. Rev. Phys. Chem. 20, 191 (1969); (c) D. Beck, in Proceedings of the International School of Physics, edited by $\mathrm{Ch}$. Schlier (Academic, New York, 1968) Course 44, p. 15; (d) R. B. Bernstein and J. T. Muckerman, Adv. Chem. Phys, 12, 389 (1967); (e) E. A. Mason and L. Monchick, ibid. 12, 329 (1967); (f) R. B. Bernstein, ibid. 10, 75 (1966); (g) H. Pauly and J. P. Toennies, Adv. At. Mol, Phys. 1, 195 (1965); (h) G. L. Pollack, Rev. Mod. Phys. 36, 748 (1964).

${ }^{4}$ See, for example, D. D. Konowalow and D. S. Zakheim, J. Chem. Phys. 57, 4375 (1972).

${ }^{5}$ See, for example, (a) J. A. Barker, R. O. Watts, J. K. Lee, T. P. Schafer, and Y. T. Lee, J. Chem. Phys. 61, 3081 (1974); (b) L. W. Bruch and I. J. MeGee, ibid. 62, 5884 (1970); (c) M. V. Bobetic and J. A. Barker, Phys. Rev. B 2, 4169 (1970); (d) J. H. Dymond and B. J. Adler, Chem. Phys. Lett. 2, 54 (1968); (e) J. A. Barker and A. Pompe, Aust. J. Chem. 21, 1683 (1968).

${ }^{6}$ H. M. Lin and R. L. Robinson, J. Chem. Phys. 62, 3727 (1970).

7(a) J. H. Dymond and B. J. Alder, J. Chem. Phys. 51, 309 (1969); (b) C. R. Mueller and J. W. Brackett, J. Chem. Phys, 40, 654 (1964).

${ }^{8}$ B. J. Jody, S. C. Saxena, V. P. S. Nain, and R. A. Aziz, Chem. Phys. 22, 53 (1977).

${ }^{9}$ (a) J. P. Toennies, W. Welz, and G. Wolf, Chem. Phys. Lett. 44, 5 (1976); (b) J. G. R. Okel and J. van de Ree, J. Chem. Phys. 64, 4259 (1971).

${ }^{10}$ For some recently refined techniques also applied to the determination of interatomic potentials, see (a) J. F. Karnicky, H. H. Reamer, and C. J. Pings, J. Chem. Phys. 64, 4592 (1976); (b) E. Bar-Ziv, ibid. 64, 4943 (1976).

${ }^{11}$ See, for example, C. Y. Ng, Y. T. Lee, and J. A. Barker, J. Chem. Phys. 61, 1996 (1974).

${ }^{12}$ M. Keil, J. T. Slankas, and A. Kuppermann, "Scattering of thermal He beams by crossed atomic and molecular beams. II. He-Ar van der Waals potential," J. Chem. Phys. (in press).

${ }^{13}$ C. H. Chen, P. E. Siska, and Y. T. Lee, J. Chem. Phys, 69, 601 (1973); see also the compendium of references therein.

${ }^{14}$ (a) K. M. Smith, A. M. Rulis, G. Scoles, R. A. Aziz, and V. Nain, J. Chem. Phys. 67, 152 (1977); (b) K. M. Smith, A. M. Rulis, G. Scoles, R. A. Aziz, and G. Duquette, ibid. 68, 2250 (1975).

${ }^{15}$ R. W. Bickes and R. B. Bernstein, J. Chem. Phys. 66, 2408 (1977).

${ }^{16}$ J. L. Dunham, Phys. Rev, 41, 721 (1932).

${ }^{17}$ G. Simons, R. G. Parr, and J. M. Finlan, J. Chem. Phys. 59, 3229 (1973).

${ }^{18}$ R. W. Bickes and R. B. Bernstein, Chem. Phys. Lett. 26, 457 (1974). The SPFD potential that we use is actually a further variant of their modifications to the SPF potential. ${ }^{17}$ The major difference lies in allowing the $c_{2 i+4}$ coefficients to be treated as parameters.

${ }^{19}$ (a) For a generalization of the Dunham series, see A. J. Thakkar, J. Chem. Phys. 62, 1693 (1975); (b) For comments on the applicability of the power series method, see C. L. Beckel, ibid. 65, 4319 (1976).

${ }^{20}$ D. H. Winicur, A. L. Moursund, W. R. Devereaux, L. R. Martin, and A. Kuppermann, J. Chem. Phys. 62, 3299 (1970); J. M. Farrar and Y. T. Lee, ibid . 67, 5492 (1972); A. Kuppermann, R. J. Gordon, and M. J. Coggiola, Faraday Discuss. Chem. Soc. 55, 145 (1973); R. W. Bickes, G. Scoles, and K. M. Smith, Can. J. Phys. 53, 435 (1975); A. M. Rulis and G. Scoles, Chem. Phys, 25, 183 (1977).

${ }^{21}$ See especially U. Buck, J. Chem. Phys. 54, 1923 (1971).
${ }^{22}$ See D. E. Freeman, K, Yoshino, and Y. Tanaka, J. Chem. Phys. 67, 3462 (1977) and earlier work by the same authors. ${ }^{23} B \equiv 2 \mu \epsilon r_{m}^{2} / \hbar^{2}$ is the "well-capacity" parameter. ${ }^{3 t}$ For He-Ar, $B \simeq 50$, a "low $-B$ " system.

${ }^{24}$ (a) R. B. Gerber and M. Shapiro, Chem. Phys. 13, 227 (1976); (b) M. Shapiro and R. B. Gerber, ibid. 13, 235 (1975); see also (c) M. Shapiro, R. B. Gerber, U. Buck, and J. Schleusener, J. Chem. Phys. 67, 3570 (1977).

${ }^{25} \mathrm{M}$. Shapiro, private communication to A. Kuppermann.

${ }^{26}$ See, however, a recent study by E. A. Colburn and A. E. Douglas, J. Chem. Phys. 65, 1741 (1976).

${ }^{27}$ (a) Y. Tanaka and K. Yoshino, J. Chem. Phys. 57, 2964 (1972); see also (b) M. V. Bobetic and J. A. Barker, J. Chem. Phys. 64, 2367 (1976).

${ }^{28}$ (a) G. Starkschall and R. G. Gordon, J. Chem. Phys. 54, 663 (1971); (b) H. L. Kramer and D. R. Herschbach, ibid. 53, 2792 (1970); (c) P. W. Langhoff and M. Karplus, ibid. 53, $233(1970)$.

${ }^{29}$ (a) Y. S. Kim and R. G. Gordon, J. Chem. Phys. 61, 1 (1974); (b) J. S. Cohen and R. T Pack, ibid. 61, 2372 (1974); (c) R. G. Gordon and Y. S. Kim, ibid. 56, 3122 (1972).

${ }^{30}$ See also (a) J. Lloyd and D. Pugh, Chem. Phys. Lett. 26, 281 (1974); (b) A. I. M. Rae, ibid. 18, 574 (1973).

${ }^{31}$ For simple models, please see (a) K. T. Tang and J. P. Toennies, J. Chem. Phys. 66, 1496 (1977); (b) J. Hepburn, G. Scoles, and R. Penco, Chem. Phys. Lett. 36, 451 (1975).

${ }^{32}$ Please see P. D. Dacre, Chem. Phys. Lett. 60, 147 (1977) for very recent results; earlier work is cited therein.

${ }^{33}$ T. J. P. O'Brien and R. B. Bernstein, J. Chem. Phys. 61, 5112 (1969).

${ }^{34}$ See also (a) A. L. J. Burgmans, J. M. Farrar, and Y. T. Lee, J. Chem. Phys. 64, 1345 (1976); (b) J. M. Parson, P. E. Siska, and Y. T. Lee, ibid. 56, 1511 (1972); (c) P. E. Siska, J. M. Parson, T. P. Schafer, and Y. T. Lee, ibid. 55, 5762 (1971).

${ }^{35}$ Our list of "common" functional forms is certainly not exhaustive; for other recently proposed forms that look promising, please see (a) G. C. Maitland and E. B. Smith, Chem. Phys. Lett. 22, 443 (1973); (b) V. H. Smith and A. J. Thakker, ibid. 17, 274 (1972).

${ }^{36}$ Other comparisons of the attributes of various potential forms may be found in, for example, (a) A. N. Dufty, G. P. Matthews, and E. B. Smith, Chem. Phys. Lett. 26, 108 (1974); (b) A. E. Sherwood and J. M. Prausnitz, J. Chem. Phys. 41, 429 (1964); (c) R. A. Aziz, ibid. 65, 490 (1976). See also Ref. 10.

${ }^{37}$ M. Klein and H. J. M. Hanley, J. Chem. Phys. 53, 4722 (1970).

${ }^{38}$ The dispersion parameters are written in reduced form as

$$
c_{2 i+4}=\frac{\mathcal{C}_{2 i+4}}{\epsilon r_{m}^{i j+4}}, \quad(i=1,2,3) \text {. }
$$

${ }^{39}$ Instead of using the constant $\delta$ of Ref. 5e to avoid spurious maxima at small separations, we replace this region of the potential with an exponentially repulsive piece; see text below. ${ }^{40}$ U. Buck and H. Pauly, Z. Phys. 208, 390 (1968).

${ }^{4 t} \mathrm{~J}$. M. Parson and Y. T. Lee, Entropie 42, 146 (1971). Modifications made to their MSV potential concern the placing of the spline points. We have generally found that the spline region can best be freed of "wiggles" for a wide variety of $\beta$, $\rho_{2}$, and $c_{2 i+4}$ values if $\rho_{1}$ is chosen as in the text.

${ }^{42}$ M. Rosen and D. R. Yennie, J. Math. Phys. 5, 1505 (1964).

${ }^{43}$ N. R. Draper and H. Smith, Applied Regression Analysis (Wiley, New York, 1966).

${ }^{44}$ See also G. Starkschall and R. G. Gordon, J. Chem. Phys. 56,2801 (1972).

${ }^{45}$ This result is different from the experience of Bickes and Bernstein. ${ }^{15}$ Also, our iterative procedure is more straightforward, requiring only one "pass" to fit the DCS. Their difficulty may be due to the different parameterization used for the SPFD potential, or to a small value of the finite-difference 
multiplier " $\triangle \mathrm{PAR}$ "; we use a value of 0.01 instead of $10^{-5}$. Perhaps a more significant difference is their use of the linearization model; we have found it best to use the nonlinear fitting algorithm ${ }^{45}$ with four or more parameters. ${ }^{12}$

${ }^{46}$ D. W. Marquardt, J. Soc. Ind. Appl。Math。11, 431 (1963).

${ }^{47}$ F. J. Smith, Physica (Utrecht) 30, 497 (1964).

${ }^{48}$ J. M. Parson, T. P. Schafer, P. E. Siska, F。 P. Tully, Y. C. Wong, and Y. T. Lee, J. Chem. Phys, 53, 3755 (1970).

${ }^{49} \mathrm{~J}$. M. Farrar, Y. T. Lee, V. V. Goldman, and M. L. Klein, Chem. Phys。 Lett. 19, 359 (1973).

${ }^{50}$ The first dispersion term may now be calculated for the rare gas pairs more accurately than $15 \%$. The higher-order terms may often be estimated theoretically to this accuracy. See, for example, (a) K. T. Tang, J. M. Norbeck, and P. R. Cer- tain, J. Chem. Phys. 64, 3063 (1976); (b) M. Alvarez-Rizzatti, and E. A. Mason, ibid. 59, 518 (1973); (c) J. T. Broussard and N. R. Kestner, ibid. 58, 3593 (1973).

${ }^{51} \mathrm{~J}, \mathrm{M}$. Farrar and $\mathrm{Y}$. T。 Lee, J. Chem. Phys。56, 5801 (1972). ${ }^{52}$ This collision energy corresponds closely with those of experiments using liquid nitrogen -cooled He beams. ${ }^{13,14}$

${ }^{53}$ Somewhat similar conclusions are expressed by D. Bassi, M. G. Dondi, F. Tommasini, F. Torello, and U. Valbusa, Phys. Rev. A 13, 584 (1976).

${ }^{54}$ Deterioration of the $\Delta \alpha_{0.95} / \alpha$ statistic by about $1 \%$ for the DCS with no data scatter is regarded as "statistically significant." It should be noted that this corresponds to an increased $\chi^{2}$ statistic of about 150; cf. Table I. 